\title{
A HIGH STABILITY, LOW NOISE RF DISTRIBUTION SYSTEM *
}

\author{
Josef Frisch, Dorel Bernstein, David Brown, Eugene Cisneros, SLAC
}

\begin{abstract}
Next generation linear colliders require high stability, low noise distribution of RF phase and timing signals. We describe a fiber-optics system that transmits phase at $357 \mathrm{MHz}$, at a $1500 \mathrm{~nm}$ wavelength, over a distance of 15 kilometers. Phase length errors in the transmission fiber are measured using the phase of the signal reflected from the fiber end. Corrections are performed by controlling the temperature of a 6-kilometer fiber spool placed in series with the main transmission fiber. This system has demonstrated a phase stability better than 10 femtoseconds per degree $\mathrm{C}$, per kilometer, an improvement of a factor of $>2000$ relative to un-stabilized fiber. This system uses standard low cost telecom fiber and components.
\end{abstract}

\section{SYSTEM OVERVIEW}

\subsection{Performance Requirements}

Table 1: Requirements for the NLC (approximate)

\begin{tabular}{|l|l|l|}
\hline Parameter & Requirement & Prototype \\
\hline Length & 15 Kilometers & 15 Kilometers \\
\hline $\begin{array}{l}\text { Noise: } 10 \mathrm{sec} \\
\text { to } 10 \mathrm{kHz}\end{array}$ & $<0.12 \mathrm{ps} \mathrm{RMS}$ & $0.25 \mathrm{ps} \mathrm{RMS}$ \\
\hline $\begin{array}{l}\text { Stability }<1 \\
\text { hour }\end{array}$ & $+/-1 \mathrm{ps}$ & $+/-0.75 \mathrm{ps}$ \\
\hline $\begin{array}{l}\text { Stability long } \\
\text { term }\end{array}$ & $+/-5 \mathrm{ps}$ & $+/-2 \mathrm{ps}$ (1 month) \\
\hline $\begin{array}{l}\text { Temperature } \\
\text { sensitivity }\end{array}$ & $<2 \times 10^{-8} / \mathrm{C}$ & $<10^{-8} / \mathrm{C}$ \\
\hline
\end{tabular}

\subsection{Technical Issues}

The most critical problem is the required long-term phase length stability of $\sim 10^{-7}$. Both copper coaxial cable and fiber optics have phase length coefficients of $\sim 10^{-}$ ${ }^{5} /{ }^{\circ} \mathrm{C}$. Obtaining long term temperature stability of $<.01$ degrees $\mathrm{C}$ would be very difficult for long (multikilometer) systems. If the distribution cables are buried in an underground trench (but not otherwise temperature controlled) summer to winter temperature variations of $+/$ $5 \mathrm{C}$ can be expected (based on temperatures measured at SLAC). This requires an improvement of a factor of $\sim 1000$ relative to unstabilized copper or fiber.

Phase noise is also a significant problem. The dispersion of fiber is typically $10 \mathrm{ps} / \mathrm{nm} / \mathrm{Km}$. This implies a required line width from the laser of $<.001 \mathrm{~nm}$ $(\sim 100 \mathrm{MHz})$. Note that slow wavelength variations are corrected by feedback. The RF electronics can also contribute significant phase noise and drift.

\footnotetext{
* Work supported by DOE contract DE-AC03-76SF00515
}

\subsection{Principal of Operation}

The phase distribution system uses single mode optical fiber operating in the minimum loss wavelength range of approximately $1500 \mathrm{~nm}$. The optical signal is amplitude modulated at the RF carrier frequency.

At the receiver end, part of the optical signal is coupled to an optical detector and used to detect the transmitted phase. The remainder is reflected from a fiber mirror and sent back to the transmitter.

At the transmitter, an optical circulator directs the reflected light to an optical detector. The RF phase measured at this detector provides a measure of the phase length of the transmission cable.

A compensating fiber cable, placed in a temperature controlled oven, is connected in series with the main transmission fiber The oven temperature is controlled in feedback to stabilize the phase of the reflected signal, and therefore the phase length of the combination of main and compensating fibers.

A detector in the transmitter is used to measure the RF phase of the transmitted signal. The RF driving the laser diode is phase controlled to stabilize the transmitted phase. This corrects for phase drifts in the transmitter diode.

The system operates in "point to point" mode: A separate transmitter / receiver pair is required to send the phase to each location. Note that is not possible to "tap" the phase signal at multiple points along the length of the transmitter fiber - spatial variation in phase length would not be compensated.

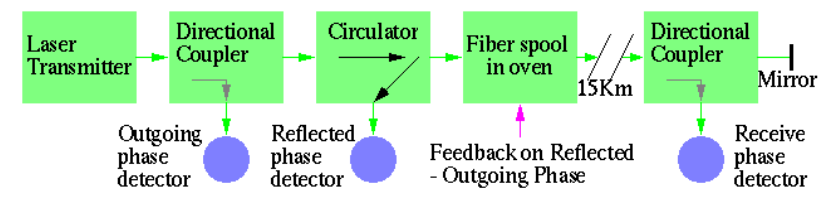

Figure 1: Conceptual Diagram

\section{PROTOTYPE SYSTEM DESIGN}

\subsection{Component Selection}

The prototype system is constructed primarily from standard telecom components. The main and compensating fibers are SMF28 single mode fiber. The transmitter is a DFB laser diode operation at $\sim 1530 \mathrm{~nm}$, directly modulated to produce the RF. The receivers are designed for OC-24 applications: approximately $1.2 \mathrm{GHz}$ bandwidth. FC/APC fiber connectors are used throughout. The final system is also expected to use standard commercial components. The NLC will require approximately 200 transmission systems, so component cost is a significant issue. 
The prototype uses "connectorized" RF components to allow easy modifications, while the final system would use surface mounted components. All of the RF and optical components are mounted in a temperaturecontrolled oven.

A Windows PC running special purpose software, with a $200 \mathrm{KHz}$ 16-bit digitizer card is used for data acquisition. The computer is installed in the same temperature controlled oven as the RF components. The long $(15 \mathrm{Km})$ spool of fiber is mounted in a control oven that simulates changes in ambient temperature.

The compensating fiber is mounted in a fast response oven. This oven is continuously cooled by a TEC cooler, and heated by a wire grid. While the time constant of the fiber spool is $\sim 1$ hour, the outer layers of fiber respond to temperature changes with a timescale of a few seconds, allowing a fairly fast feedback speed.

\subsection{Prototype System Operation}

The system RF is operated at $357 \mathrm{MHz}$, chosen as the $1 / 32$ sub harmonic of the NLC $11.424 \mathrm{GHz}$ main RF frequency. Measurements indicate that the phase detection at the relatively low frequency $357 \mathrm{MHz}$ is not a significant noise or drift source.

The laser diode, and its RF modulation are pulsed with an 80 -microsecond pulse at a $3125 \mathrm{~Hz}$ repetition rate. This allows the phase detector to be multiplexed between the outgoing, fiber end, and reflected phases. The received RF signals are down mixed to a $25 \mathrm{KHz} \mathrm{IF}$, and then digitized at $200 \mathrm{KHz}$. The phase is measured digitally on each pulse (at $3125 \mathrm{~Hz}$ ), and averaged over a $10 \mathrm{~Hz}$ feedback cycle.

The fiber length feedback output directly controls the heater grid in the compensation fiber oven. The outgoing phase feedback controls an RF phase shifter on the drive for the laser diode.

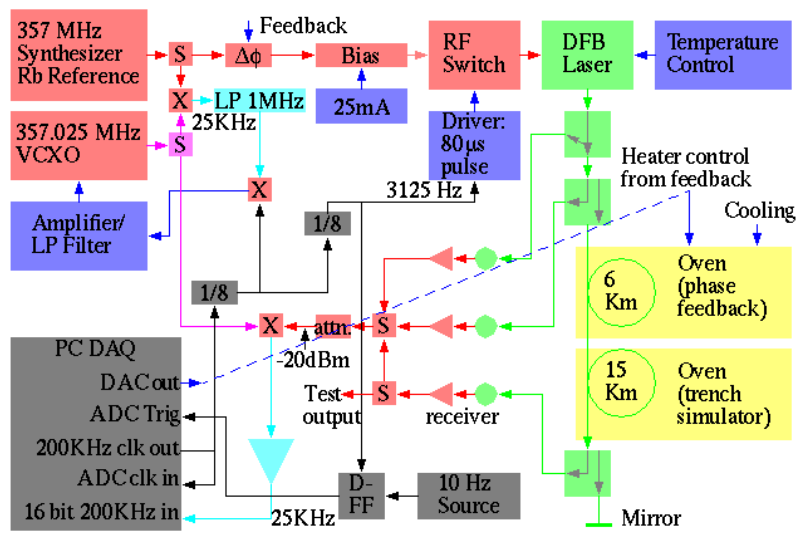

Figure 2: System Block Diagram

\subsection{Independent Phase Measurement}

The data acquisition system monitors the phase measured at the end of the fiber, but does not use that information in feedback. An independent phase measurement is performed by directly mixing the test output signal (Fig 2) with the RF source output. This signal is available for high frequency noise measurements and for cross checking low frequency measurements.

The test output signal is narrow band filtered with a $\sim 100 \mathrm{~Hz}$ bandwidth VCXO phase locked loop to reduce broadband noise. A filter of this type would be also be used on the output of a production system.

\section{PERFORMANCE MEASUREMENTS}

\subsection{Long Term Drift Measurements}

The prototype was operated for a month with the transmission fiber set to a constant temperature. The direct data acquisition system readout and the independent phase measurement show that the drift is within $+/-2$ picoseconds. Note that the disagreement between the two is believed due to drift in the (less stable) analog readout. The NLC RF system can correct the measured long-term drift in the phase distribution system with beam-based measurements [1].

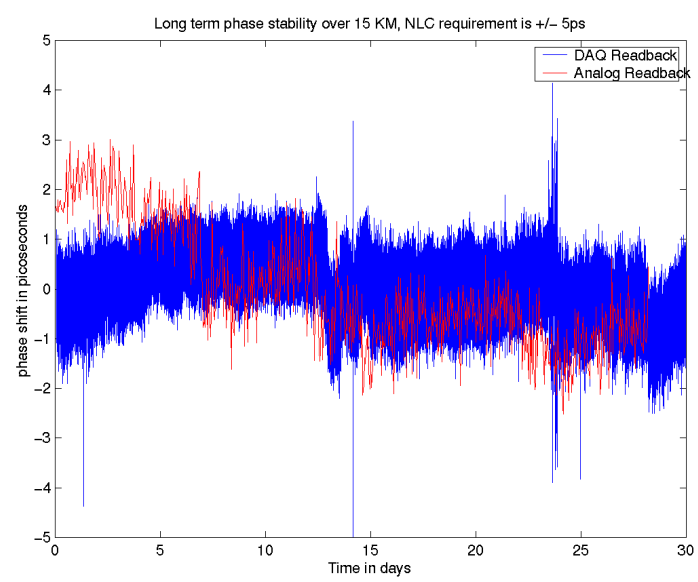

Figure 3: Drift $<+/-2$ ps over 1 month for a $15 \mathrm{Km}$ fiber

\subsection{Temperature Sensitivity Measurements.}

The system phase stability was tested for a $10^{\circ} \mathrm{C}$ temperature change of the distribution fiber. The timing shift was $\sim 1 \mathrm{ps}$, a factor of 5000 smaller than for uncompensated fiber. This meets the NLC requirement.
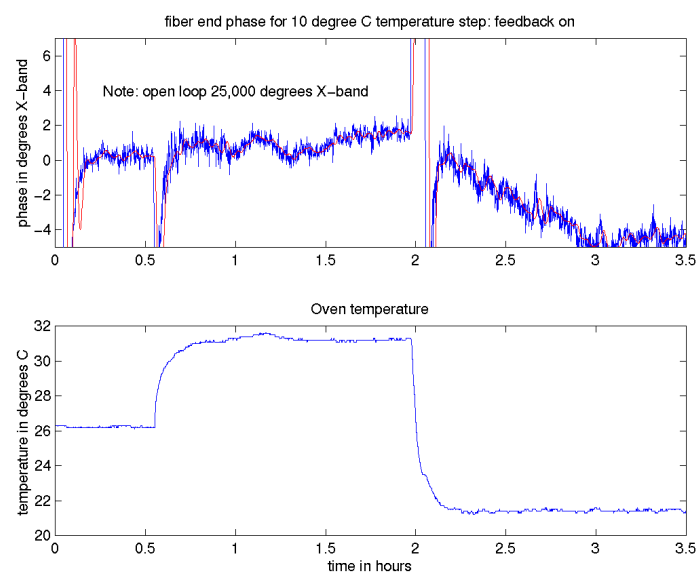

Figure 4: Phase stability: transmission fiber $\Delta \mathrm{T}=10^{\circ} \mathrm{C}$. 


\subsection{Phase Noise Measurements}

The signal from the test output mixed with the main RF is used to measure the phase noise of the transmission system. The total noise integrated from $1 \mathrm{~Hz}$ to $10 \mathrm{KHz}$ is $0.25 \mathrm{ps}$, approximately a factor of 2 worse than the NLC requirement. Figure 5 shows the phase noise in octave blocks. Note that the vertical scale is uncalibrated.

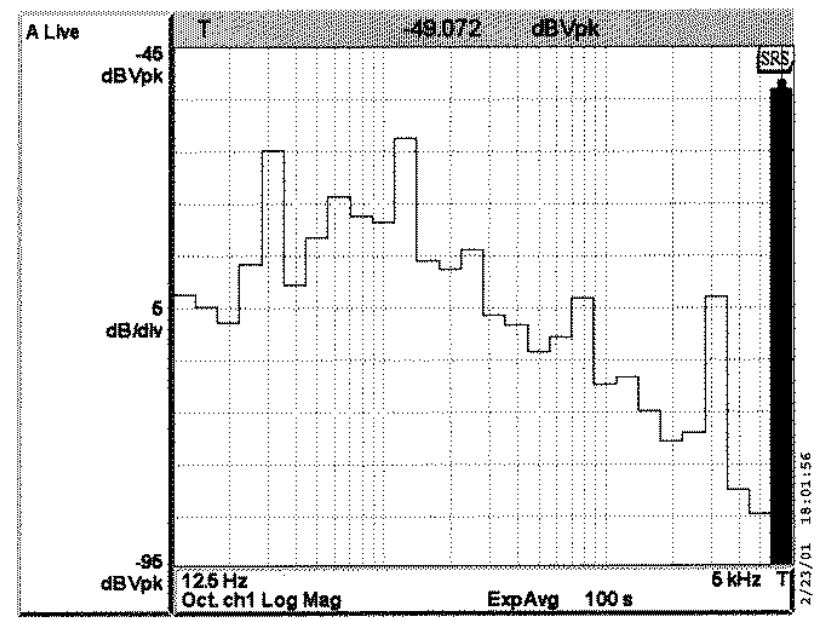

Figure 5: Integrated phase noise is $0.25 \mathrm{ps}$.

\subsection{Phase Noise Improvement Plans}

The phase noise spectrum from the transmission fiber is fairly flat with frequency. A series of 2 phase locked loops $(300 \mathrm{~Hz}$ bandwidth, and $100 \mathrm{~Hz}$ bandwidth) are used to limit the bandwidth and reduce the integrated noise. The feedback loops for the oscillators have not been optimized to reduce the overall system noise, and some improvement may be possible.

Wavelength noise in the laser is believed to be a significant source of phase noise. A short-term line width of $100 \mathrm{MHz}$ could be responsible for the observe noise. A lower noise laser is being installed on the system.

\subsection{Next Generation Prototype}

A new prototype is being developed which will more closely resemble a production system. The following changes are planned:

- The DFB laser diode will be replaced with a newer, higher performance telecommunications part. This should provide lower noise, and allow operation at $714 \mathrm{MHz}$.

- The SMA connector RF components will be replaced with surface mount components.

- A fiber stretcher will be added to provide higher bandwidth feedback.

- The Windows data acquisition system will be replaced with a micro controller based system.

\section{STATUS AND CONCLUSIONS}

The phase transmission system has demonstrated the noise and temperature sensitivity performance required for the NLC. The phase noise is a factor of 2 worse than required, but is expected to be reduced with a lower noise laser diode.

The prototype has demonstrated $+/-2$ picosecond timing stability in a 15 kilometer link for one month and with $10^{\circ} \mathrm{C}$ temperature variations of the distribution fiber. The system design uses low cost telecommunications components.

\section{REFERENCES}

[1] J. Frisch, D. Brown, E. Cisneros, "The RF Phase and Timing Distribution System for the NLC", SLAC PUB 8578 August 2000 